\title{
A new method for vehicles detection and tracking using information and image processing
}

\author{
Mazouzi Amine' ${ }^{1}$ Kerfa Djoudi ${ }^{2}$, Ismail Rakip Karas ${ }^{3}$ \\ ${ }^{1}$ Nationale Institute of Telecommunications ICT of Oran Algeria, Oran, Algeria \\ ${ }^{2}$ Polytechnic National School of Oran Maurice Audin, Oran, Algeria \\ ${ }^{3}$ Faculty of Engineering, Computer Engineering Department, Demir Celik Campus, Karabuk, Turkey
}

\begin{tabular}{l} 
Article Info \\
\hline Article history: \\
Received Sep 2, 2020 \\
Revised Apr 12, 2021 \\
Accepted May 2, 2021 \\
\hline Keywords: \\
Image processing \\
Information processing \\
Labeling \\
Tracking \\
Vehicles detection
\end{tabular}

\section{Corresponding Author:}

Mazouzi Amine

Nationale Institute of Telecommunications ICT

POB 1518, El Mnouar, Es Senia street, Oran, Algeria

Email: amazouzi@inttic.dz

\begin{abstract}
In this article, a new method of vehicles detecting and tracking is presented: A thresholding followed by a mathematical morphology treatment are used. The tracking phase uses the information about a vehicle. An original labeling is proposed in this article. It helps to reduce some artefacts that occur at the detection level. The main contribution of this article lies in the possibility of merging information of low level (detection) and high level (tracking). In other words, it is shown that many artefacts resulting from image processing (low level) can be detected, and eliminated thanks to the information contained in the labeling (high level). The proposed method has been tested on many video sequences and examples are given illustrating the merits of our approach.
\end{abstract}

This is an open access article under the CC BY-SA license.

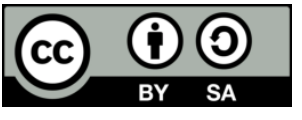

\section{INTRODUCTION}

The intelligent transportation system [1]-[5] are axis of great importance and topicality. It not only helps to smooth the flow of vehicles but also reduces the number of accidents. In this context, the detection and tracking of vehicles are of crucial importance, which explains the large number of works [6]-[10].

It is known that detection methods [11] based on a motion approach are of less complexity and are therefore more suitable for their use in real time. Most of these methods [12]-[16] of detection is performed by subtraction into two successive images and binarization. It appears that this approach although fast, suffers from many artifacts.

Many works have been done to reduce the problem of artefacts. In [17] the authors propose a texture mixture of Gauss in order to distinguish the background of the image, facilitating the detection of moving vehicles. This approach is better adapted within the framework of a moving camera. The work that was done in [18] exploits the principle of Mahalanobis distance in order to compute the background image allowing by the end to select moving vehicles, however the computational cost make this approach less effective in real time issue.

Wang et al. [19] used the notions of extended optical flux and Eigen space to differentiate static parts from moving parts. This method has a significant time cost. Chen and Zhang [20] are designed an approach regroups of the following phases: the implementation of the difference between three successive images, followed by the use of artifacts filtering, then the application of a grouping operation of the pixels and finally the exploitation of mathematical morphology and connectivity analysis process. 
The detection/tracking approaches that are based on motion study are very fast, so they are exploited in real time. However, these approaches have the disadvantage of many artifacts [21], [22]. Morphological transformation is done to deal with the problems of artifacts, but remains unsuccessful. The following Figure 1 illustrates the limits of the implementation of the thresholding phase, binarization and mathematical morphology. We observe in Figure 1(c) the subdivision of objects (see circle). In this article, the proposed method uses a motion based approach for detection. The set of artifacts generated have been reduced using a procedure of tracking 'labeling'.

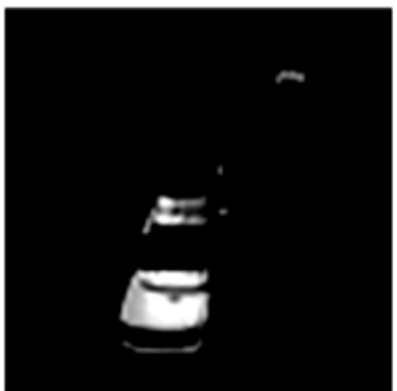

(a)

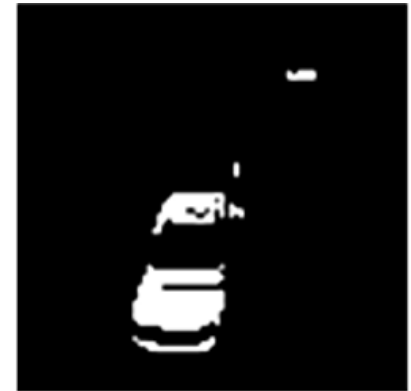

(b)

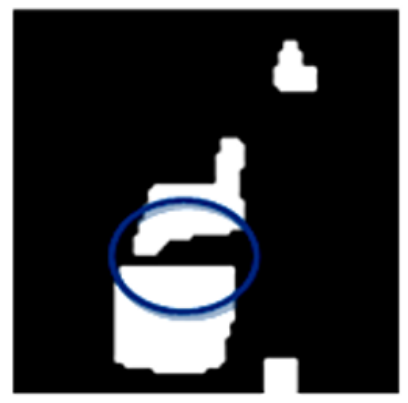

(c)

Figure 1. Image processing limits; (a) thresholding, (b) binarization, (c) mathematical morphology

\section{PROPOSED METHOD}

Our method proposed is subdivided into two levels: the low level (image processing) and the high level (information processing).

\subsection{Image processing}

The detection is achieved by the difference between two successive images, followed by a binarization. Artifacts can appear (small spots due to brightness variations). Treatment using a morphological transformation is performed. As shown in Figure 2 Each vehicle localized is registered in a Rect rectangle defined by:

$\mathrm{x}_{\mathrm{h}} \quad$ : is the highest pixel position of the vehicle.

$\mathrm{x}_{\mathrm{l}} \quad:$ is the lowest pixel position of the vehicle.

$\mathrm{y}_{\mathrm{r}} \quad$ : is the rightmost pixel position of the vehicle.

yl : is the leftmost pixel position of the vehicle.

$c_{i}, c_{j}:$ are the geometric center coordinates of the rectangle containing the vehicle.

A vehicle has been characterized by this rectangle Rect and the matrix $\mathrm{Mg}$ of gray level (gray level of the pixels contained in Rect). A vehicle detected at time $t+1$ is searched in the previous image $t$ on a search area [23], [24] defined from the vehicle's geometry center of the image $t+1$.

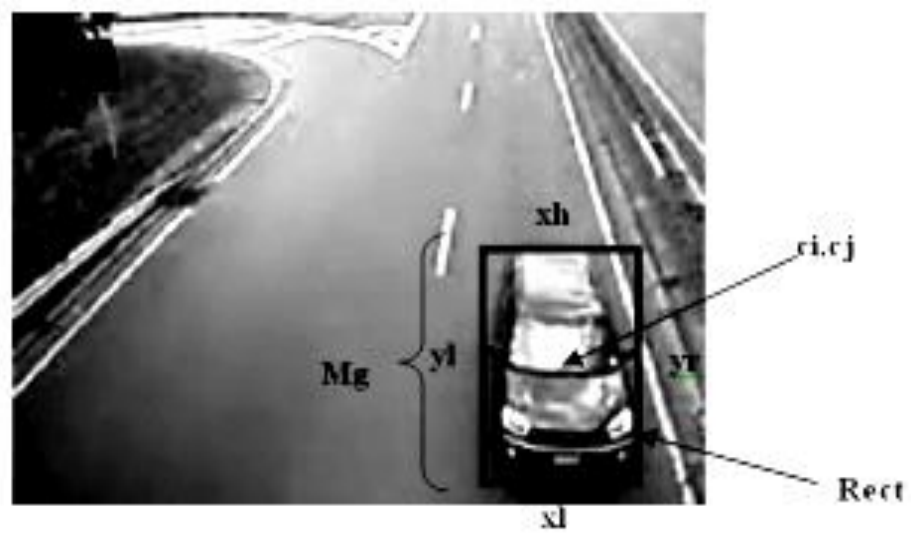

Figure 2. Parameters that represent a vehicle 
Where more than one vehicle is in the search area, we must proceed with the likenhood procedure to ensure the correspondence.

Noting that in our work, the search area is defined in the images by the sizes: $(-5,+5),(-10,+10)$ and $(-20,+20)$ from top to bottom of the image. For this, we exploit the histograms in gray level [25] calculated from the matrix $\mathrm{Mg}$ of each vehicle. In Figure 3 an illustrative example is given. Assuming that we want to establish a correspondence between the vehicles of the moment $t+1$ which is at the top of the image in Rect of Figure 3(b) and the vehicles located at time t.

After inserting the search area at time $t$ see Figure 3(a) in discontinues lines, we found two vehicles represented by these geometric centers that are included in this area. The next phase is the modeling of the $\mathrm{Mg}$ gray level histograms for this vehicle and the two others belonging to the search area, these histograms are used to calculate the Vehicle Surface area.

$$
\text { Area véhiculei }=\sum \text { Hist }_{n g} \mathrm{~V}_{\mathrm{i}}^{2}(\mathrm{k})
$$

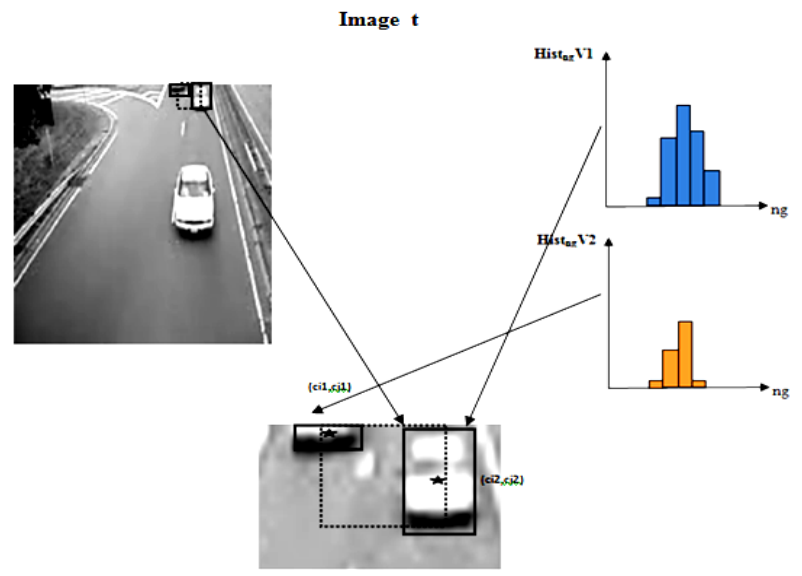

(a)

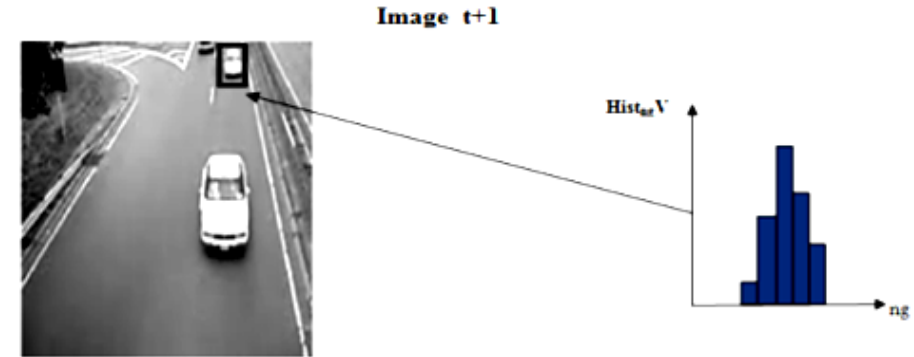

(b)

Figure 3. Illustrative example; (a) image of the moment $t$ and the gray level histogram for the two localized vehicles, (b) image of the moment $t+1$ and the gray level histogram for the vehicle to follow

Finally, a decision is made, which is based on a comparison between the histogram surfaces of the two vehicles that are at time $t$ and that of vehicle of time $t+1$. This decision was made by calculating the distances between these surfaces, by selecting what has the smallest distance according to the following rule:

Vehicle selected $=$ min distance [(Area VR, Area V1) and (Area VR, Area V2)]

Area VR : area of a search vehicle.

Area V1 : area of the first vehicle found.

Area V2 : area of the second vehicle found.

\subsection{Information processing "Labeling"}

For each vehicle detected at time $t$, it is attributed a label defined by a doublet $\left(a_{t}, c_{t}\right)$ and $\left(a_{t+1}, c_{t+1}\right)$ at time $t+1$. The detection system scans the image from top to bottom and from left to right. In the image $t$, 
the first vehicle detected $a_{t}=1$, the second vehicle detected will have $a_{t}=2$. So the number $a_{t}$ gives the order of appearance of the vehicle in the image $t$. The digit $c_{t}$ indicates the correspondence, the vehicle of label $\left(a_{t+1}\right.$, $c_{t+1}$ ) appeared at the $a_{t+1}$ time in the image $t+1$ and corresponds to the vehicle which appeared at the $c_{t+1}$ time in the previous picture $t$.

In Figure 4(a), we have two images t, which represent an empty road and a road has three vehicles carrying the labels $(1,1),(2,2)$ and $(3,3)$. While in Figure 4(b), we can see two images $t+1$, which represent a road to a vehicle labeled by $(1,0)$ and another to three vehicles labeled by $(1,1),(2,2)$ and $(3.3)$. In the images $\mathrm{t}+1$, the label $(1,0)$ indicates that there is a vehicle that has just appeared as a new object and that this vehicle does not appear in the image $t$, while the pairs $(1,1),(2,2)$ and $(3,3)$ mean that there are three vehicles in the image $t+1$ and that it is vehicles looks like the same vehicles labeled by $(1,1),(2,2)$ and $(3,3)$.
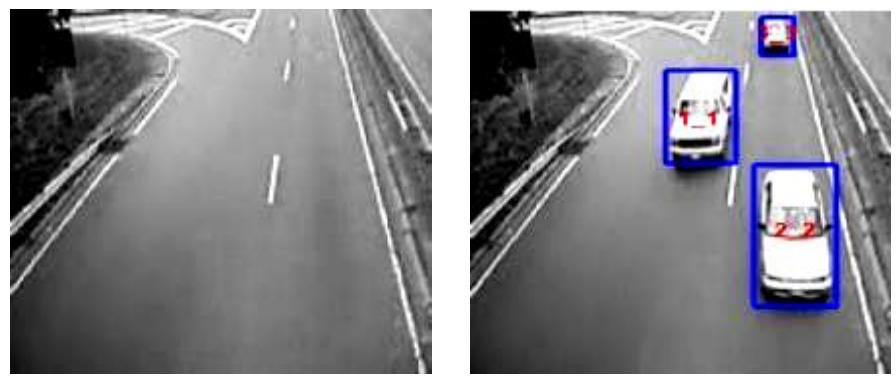

(a)
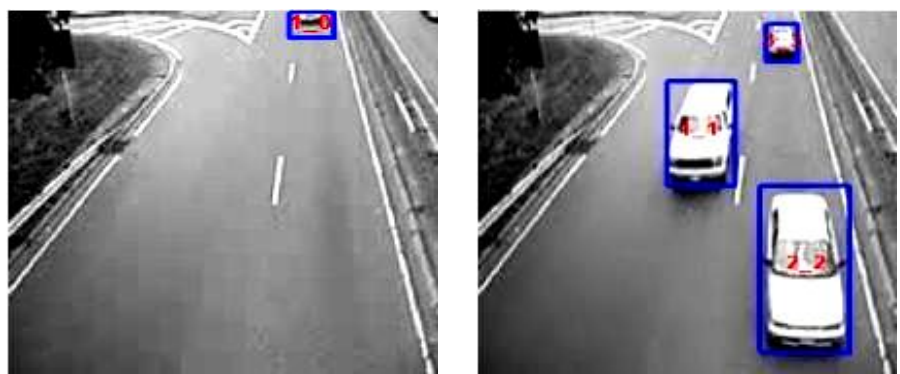

(b)

Figure 4. Principle of labels; (a) images t, (b) image $t+1$

\subsection{Discussion and ascertainment}

In the previous section, we discussed the basic principle of labeling. Now we want to develop this principle. A simple analysis shows that for a given image:

a. $\operatorname{Max}\left(\mathrm{a}_{\mathrm{t}}\right)$ is the total number of vehicles detected at time $\mathrm{t}$.

b. If at time $t+1$, we observe that one of the vehicles carries a label $c_{t+1}=0$, then the number of vehicles at time $\mathrm{t}+1$ becomes $\operatorname{Max}\left(\mathrm{a}_{\mathrm{t}}\right)+1$.

c. If $\max \left(a_{t+1}\right)=\max \left(a_{t}\right)-1$, then there is a disappearance of a vehicle in the image $t+1$.

Since the low level of processing, which is particularly based on thresholding and mathematical morphology, generates a lot of errors, these causes: the duplication of objects, the bad appearance, and consequently reflects on the rules described above. For example, if the same vehicle of the instant $t$ will be subdivided into two objects at the instant $t+1$, in this case we can arrive in situations where these two objects will generate a problem of increase of vehicles number at time $t+1$.

Another very important example is that one can have a situation where a vehicle already appeared, will be detected as a new object. In order to rise to these problems, a correction approach that is based on rules that can be summarized by the following points:

a. The phenomenon of appearance is only for a new vehicle that is in the area of appearance (the area farthest from the camera).

b. On the other hand, the phenomenon of disappearance is defined if a vehicle of moment $t$ appears in the zone of disappearance (the zone closest to the camera) and that this object does not appear at time $\mathrm{t}+1$.

In this article, we considered the simple case of a one-way road (from top to bottom or from right to left). We performed during an appearance of a vehicle the value 0 to $\mathrm{c}$. The addition of these rules allows a clear improvement for our detection/tracking method. Our approach is to apply a correction procedure, it is given by the following algorithm.

A new method for vehicles detection and tracking using information and image processing (Mazouzi Amine) 
Begin

a. Read of image.

b. An enlargement of the search area built around vehicles with labelling errors $\left(c_{t}=0\right.$ and this vehicle does not belong to the area of appearance).

c. Repeat the operation of the terrain geometry and the mapping.

d. Assign labels for vehicles with labelling errors.

e. Group objects that have the same label and (if they exist) in the same object.

f. Test the labels of the vehicles again and redo the abovementioned spots for the case of incorrect "label error" detections.

g. Stop as soon as you get a good detection.

End

\section{EVALUATION OF OUR METHOD AND RESULTS}

If we try to make an evaluation of our approach compared to the existing works, we can notice that this approach can be applied in real time compared to the different methods which are based on the artificial intelligence [26], [27]. In addition, our method is based on a principle of fusion between the aspect of image processing and information processing 'labels' which gives an advantage over conventional methods based on the aspect of image processing [28], [29].

In the Figure 5 that follows, four types of video sequences: two in road (the traffic flow from top to bottom of image), the third one inside a tunnel and the last one is in road (the traffic flow from right to left of image). We can observe a clear improvement of the detection/tracking procedure and this because of the implementation of the correction procedure. In these examples, we have tried to correct several errors, among these corrections the grouping of objects that carry the same labels and the deletion of objects that have labels of $c_{t}=0$, and which are outside the area of appearance. The modification of the objects labels which carry labels $c_{t}=0$ and which are outside the zone of appearance.
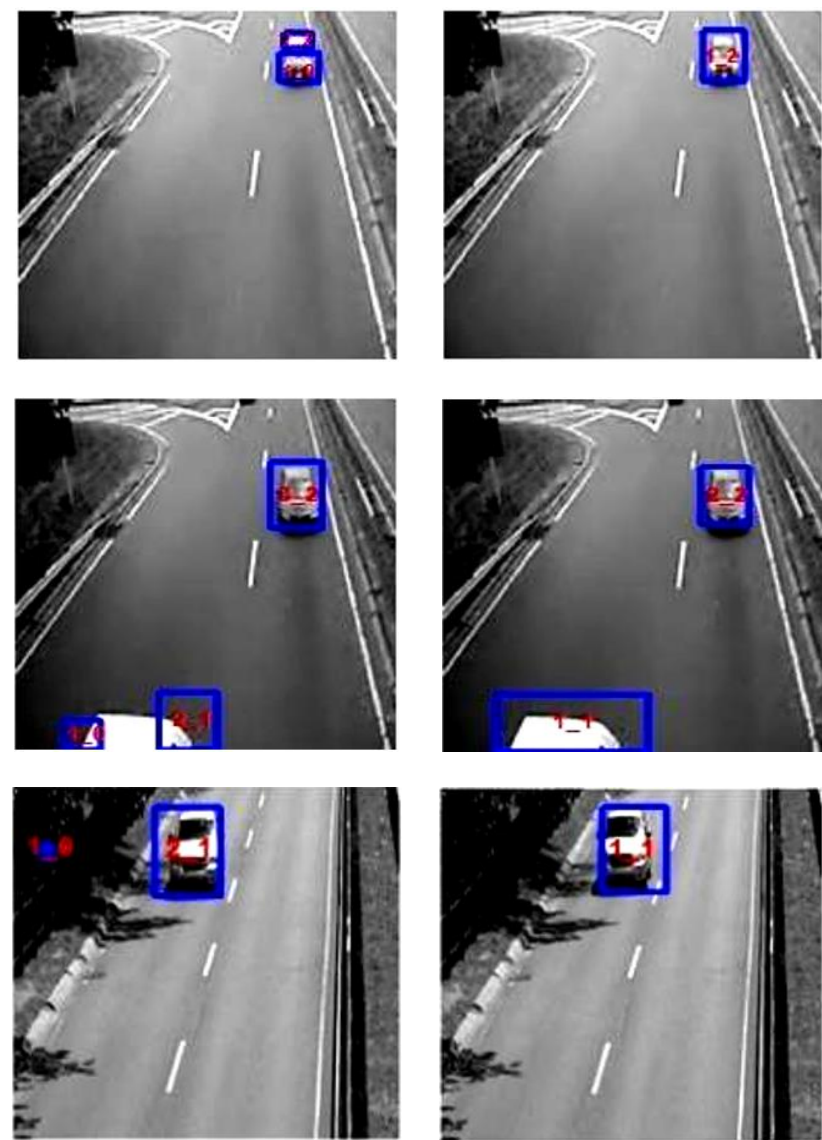

(a)

(b)

Figure 5. Detection/tracking results; (a) without the correction phase, (b) with the correction phase (continue) 

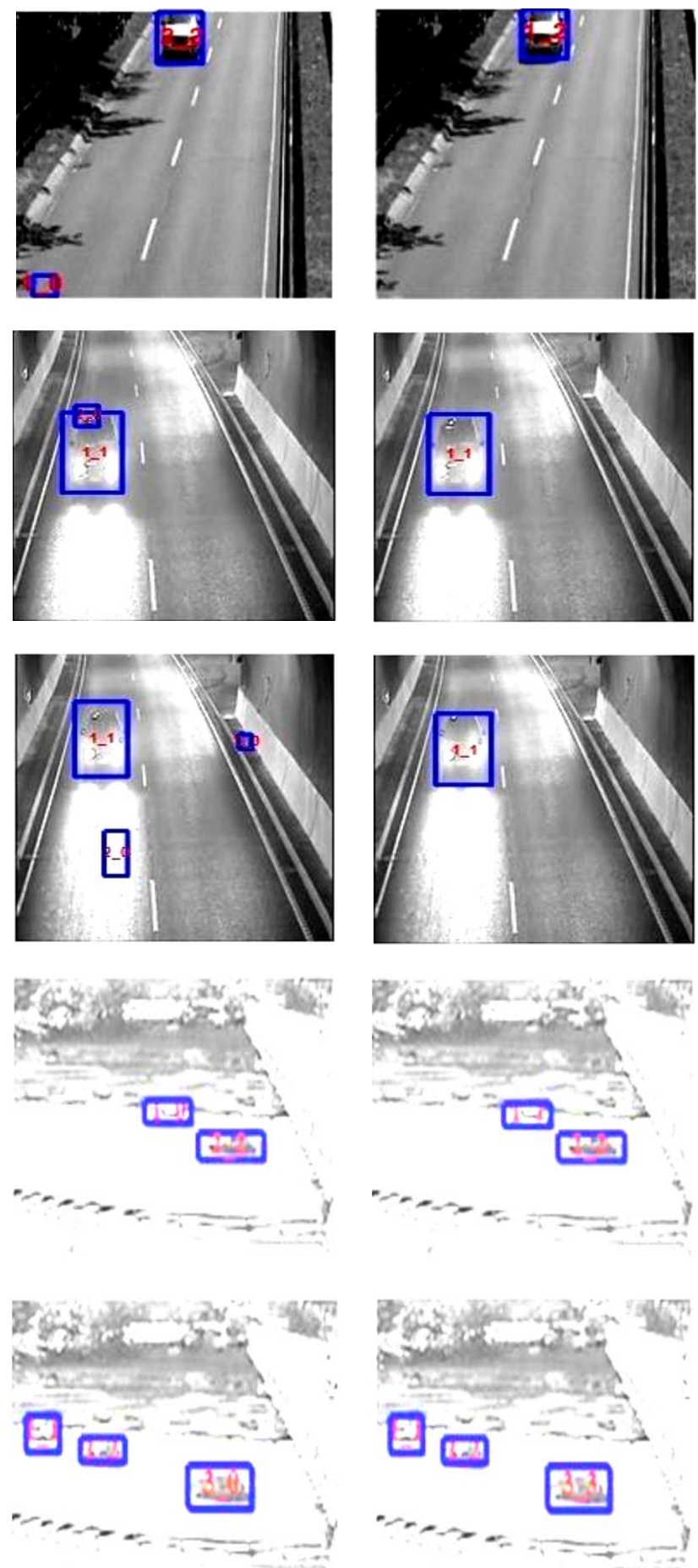

(a)

(b)

Figure 5. Detection/tracking results; (a) without the correction phase, (b) with the correction phase

\section{CONCLUSION}

In this article, a method based on two levels of processing, namely image processing and information processing, has been presented to detect and track vehicles in video sequences. Our contribution is to add a correction loop by exploiting the level of information processing which is based mainly on the principle of labeling, the latter showed its effectiveness in order to enhance the results of the vehicles detection especially in the case of the subdivision of objects, and in the presence of artifacts. The perspective work is concentred around the traffic road modelization, using a transfert matrix and the labels management. 


\section{REFERENCES}

[1] K. Poorani, A. Sharmila, and G. Sujithara, "IOT Based live streaming of vehicle, position accident prevention and detection system," International Journal of Recent Trends in Engineering \& Research (IJRTER), vol. 3, pp. 52-55, 2017.

[2] A. J. Samuel and S. Sebastian, "An algorithm for IoT based vehicle verification systemusing RFID," International Journal of Electrical and Computer Engineering (IJECE), vol. 9, no. 5, pp. 3751-3758, 2019, doi: 10.11591/ijece.v9i5.pp3751-3758.

[3] A. Mazouzi and M. F. Bel Bachir, "Enhancement of the Detection for Intelligent Vehicle Systems-Case Rain/Snow," International Review of Automatic Control (IREACO), vol. 10, no. 2, 2017, doi: 10.15866/ireaco.v10i2.8242.

[4] M. Amine and K. Djoudi, "Vehicles detection using the MLP and the correlation measurement," 2019 International Conference on Advanced Electrical Engineering (ICAEE), 2019, pp. 1-5, doi: 10.1109/ICAEE47123.2019.9015144.

[5] V. S. Padilla, R. A. Ponguillo, A. A. Abad, and L. E. Salas, "Cyber-physical system based on image recognition toimprove traffic flow A case study," International Journal of Electrical and Computer Engineering (IJECE), vol. 10, no. 5, pp. 5217-5226, 2020, doi: 10.11591/ijece.v10i5.pp5217-5226.

[6] P. A. Targe and M. P. Satone, "VANET based Real-Time Intelligent Transportation System," International Journal of Computer Applications, vol. 145, no. 4, pp. 34-38, 2016, doi: 10.5120/ijca2016910582.

[7] H. Dahhou et al., "Design and Implementation Intelligent Adaptive Front-lightingSystem of Automobile using Digital Technology on Arduinoboard," International Journal of Electrical and Computer Engineering (IJECE), vol. 8, no. 1, pp. 521-529, doi: 10.11591/ijece.v8i1.pp521-529.

[8] H. M. Ali and Z. S. Alwan, "Car Accident Detection and Notification System Using Smartphone," International Journal of Computer Science and Mobile Computing, vol. 4, no. 4, pp. 620-635, 2015.

[9] I. Ahmad, R. Md. Noor, A. Ihsan, and M. A. Qureshi, "The Role of Vehicular Cloud Computingin Road Traffic Management: A Survey," International conference on future intelligent vehicular technologies, 2017, pp. 123-131, doi: 10.1007/978-3-319-51207-5_12

[10] M. D. Kumar HSDK, S. Gupta, S. Kumar, and S. Srivastava, "Accident Detection and Reporting System Using GPS and GSM Module," Journal of Emerging Technologies and Innovative Research (JETIR), vol. 2, no. 5, pp. 1433-1436, 2015.

[11] D. Kerfa and M. F. Belbachir, "An Efficient Real Time Moving Object Detection Scheme Using Diamond Search Algorithm and Mathematical Morphology," International Review on Computers and Software (IRECOS), vol. 9, no. 05, 2014

[12] Y. Ivanov, A. Bobick, and J. Liu, "Fast lighting independent background subtraction," Proceedings 1998 IEEE Workshop on Visual Surveillance, 1998, pp. 49-55, doi: 10.1109/WVS.1998.646020.

[13] P. Bhaskar and S. P. Yong, "image processing based vehicle detection and tracking method," 2014 International Conference on Computer and Information Sciences (ICCOINS), 2014, pp. 1-5, doi: 10.1109/ICCOINS.2014.6868357.

[14] S. Gupte, O. Masoud, R. F. K. Martin, and N. P. Papanikolopoulos, "Detection and Classification of Vehicles," IEEE Transactions on Intelligent Transportation Systems, vol. 3, no. 1, pp. 37-47, 2002, doi: 10.1109/6979.994794.

[15] B. Pawar, V. T. Humbe, and L. Kundnani, "Morphology based moving vehicle detection," 2017 International Conference on Big Data Analytics and Computational Intelligence (ICBDAC), 2017, pp. 217-223, doi: 10.1109/ICBDACI.2017.8070837.

[16] B. Alshaqaqi, M. Boumehed, A. Ouamri, and M. Keche, "Implementation of distance and speed measurementalgorithms for the development of an automatic traffic regulation system," International Review on Computers and Software (IRECOS), vol. 7, no. 6, pp. 2804-2809, 2012.

[17] C. Stauffer and W. E. L. Grimson, "Adaptive background mixture models for real-time tracking," Proceedings. 1999 IEEE computer society conference on computer vision and pattern recognition (Cat. No PR00149), vol. 2, 1999, pp. 246-252, doi: 10.1109/CVPR.1999.784637.

[18] M. Tsuchikawa, A. Sato, H. Koike, and A. Tomono, "A moving-object extraction method robust against illumination level changes for a pedestrian counting system," Proceedings of International Symposium on Computer Vision - ISCV, 1995, pp. 563-568, doi: 10.1109/ISCV.1995.477061.

[19] J. Wang, G. Bebis, and R. Miller, "Overtaking Vehicle Detection Using Dynamic and Quasi-Static Background Modeling," 2005 IEEE Computer Society Conference on Computer Vision and Pattern Recognition (CVPR'05) Workshops, 2005, pp. 64-64, doi: 10.1109/CVPR.2005.506.

[20] C. Chen and X. Zhang, "Moving Vehicle Detection Based on Union of Three-Frame Difference," Advances in Electronic Engineering, Communication and Management vol. 2, vol. 140, 2012, pp. 459-464, doi: 10.1007/978-3642-27296-7_71.

[21] V. Rekhaa and K. Natarajan, "Foreground algorithms for detection and extraction of an objectin multimedia," International Journal of Electrical and Computer Engineering (IJECE), vol. 10, no. 2, pp. 1849-1858, 2020, doi: 10.11591/ijece.v10i2.pp1849-1858.

[22] M. Heikkila and M. Pietikainen, "A Texture-Based Method for Modeling the Background and Detecting Moving Objects," IEEE Transactions on Pattern Analysis and Machine Intelligence, vol. 28, no. 4, pp. 657-662, 2006, doi: 10.1109/TPAMI.2006.68.

[23] Jp. Dhanalakshmi and A. E. Sam Leni," Instance vehicle monitoring and trucking with internet of thinks usingarduino," International Journal on Smart Sensing and Intelligent Systems, vol. 2017, no. 4, pp. 123-135, 2017, doi: 10.21307/ijssis-2017-240. 
[24] A. P. Kulkarni and V. P. Baligar, "Real Time Vehicle Detection, Tracking and Counting Using Raspberry-Pi," 2020 2nd International Conference on Innovative Mechanisms for Industry Applications (ICIMIA), 2020, pp. 603-607, doi: 10.1109/ICIMIA48430.2020.9074944.

[25] J. Bossu, N. Hautière and J. P. Tarel, "Rain or Snow Detection in Image Sequences through useof a Histogram of orientation of Streaks," International journal of Computer Vision, vol. 93, no. 3, pp. 348-367, 2011, doi: 10.1007/s11263-011-0421-7

[26] A. Mohamed, A. Issam, B. Mohamed, and B. Abdellatif, "Real-time detection of vehicles using the Haar likef eatures and artificial neuron networks," Procedia Computer Science, vol. 73, pp. 24-31, 2015, doi: 10.1016/j.procs.2015.12.044.

[27] S. K. Reddy Mallidi and V. V. Vineela," IOT based smart vehicle monitoring system," International Journal of Advanced Research in Computer Science, vol. 9, no. 2, pp. 738-741, 2018, doi: 10.26483/ijarcs.v9i2.5870

[28] Y. Yang, Q. Zhang, P. Wang, X. Hu, and N. Wu, "Moving Object Detection for Dynamic Background Scenes Based on Spatiotemporal Model," Hindawi Advances in Multimedia, vol. 2017, 2017, Art. no. 5179013, doi: $10.1155 / 2017 / 5179013$.

[29] S. Sharma and S. Sebastian, "IoT based car accident detection and notification algorithm general road accident," International Journal of Electrical and Computer Engineering (IJECE), vol. 9, no. 5, pp. 4020-4026, 2019, doi: 10.11591/ijece.v9i5.pp4020-4026.

\section{BIOGRAPHIES OF AUTHORS}

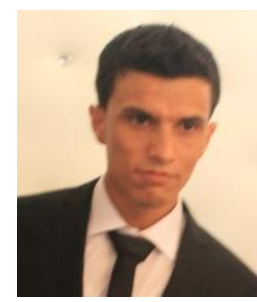

Amine Mazouzi received the degree of electronic Engineer in Electronic Engineering from the University of Sciences and Technology of Oran Mohamed Boudiaf (USTO-MB) Oran - Algeria, in 2006, the Magister degree in National Institute of telecommunications and information technologies and Communication, Oran, Algeria in 2010, and the science doctorate from the USTO-MB in 2019. Since 2014 he is at the National Institute of telecommunications and information technologies and communication, Oran-Algeria as a permanent assistant professor. $\mathrm{He}$ is interested in intelligent transportations systems, fuzzy systems, and image processing.

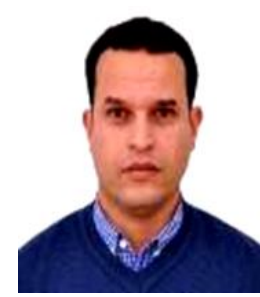

Djoudi Kerfa received the Master and Doctorate degrees from the University of Sciences and Technology of Oran, Algeria in respectively in 2009 and 2015. Since 2016, member of the laboratory CaSiCCE. In January of 2016, he joined the Department of Electrical Engineering, Polytechnic National School of Oran Maurice AUDIN, Algeria as Senior lecturer. His research interests include Image/Video compression, Image/Video post processing, Chip Architectures, technical measurement, legal metrology and control industrial processes and instrumentation.

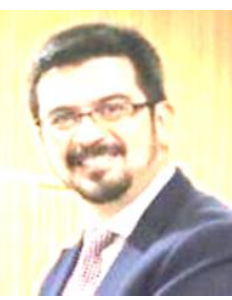

Ismail Rakip Karas is a Professor of Computer Engineering Department and Head of 3DGeoInformatics Research Group at Karabuk University, Turkey. He received his BSc degree from Selcuk University, MSc degree from Gebze Institute of Technology, and $\mathrm{PhD}$ degree from GIS and Remote Sensing program of Yildiz Technical University, in 1997, 2001 and 2007 respectively, three of them from Geomatics Engineering Department. In 2002, he involved in a GIS project at Forest Engineering Department, Oregon State University, USA. During the various periods of 2010, 2011, and 2014, he was a Visiting Researcher in 3D GIS Research Lab, Faculty of Geoinformation Science and Engineering, UniversitiTeknologi Malaysia. Between 2000-2009, he was a Research Assistant at Geomatics Engineering Department of Gebze Institute of Technology. Since 2009, he has been in Karabuk University and taught undergraduate and graduate classes in Geoinformation and Computer Sciences. Prof Dr Ismail Rakip Karas is the Member of Executive Committee in CAYDAG-TUBITAK (A Research Group of The Scientific and Technological Research Council of Turkey). He has also carried out administrative duties such as Head of Computer Science Division of Department, Director of Safranbolu Vocational School of Karabuk University. Currently, he is the Dean of Safranbolu Fine Art and Design Faculty in same university. As a keynote speaker, Prof Karas has been invited to the different high level conferences. He is the author of more than a hundred international and Turkish publications and papers on various areas of Geoinformation Science. $\mathrm{He}$ has organized and chaired some important conferences and workshops such as GeoAdvances 2017. He is in the editorial board of "International Journal of Geo-Spatial Knowledge and Intelligence", "American Journal of Geographic Information System", and some others. He has involved in Tubitak, EU, and other national and international projects and got various awards and supports during his research activities. 